\title{
Metabolic Profiling of Early and Late Recurrent Pancreatic Ductal Adenocarcinoma Using Patient-Derived Organoid Cultures
}

\author{
Lukas M. Braun ${ }^{1,2,3,+}\left(\mathbb{D}\right.$, Simon Lagies ${ }^{1,3,4,+}$ (D) Rhena F. U. Klar ${ }^{5,6}$, Saskia Hussung ${ }^{5,7}$, \\ Ralph M. Fritsch $5,6,7,8,9$, Bernd Kammerer $1,4,10, *$ and Uwe A. Wittel ${ }^{2, *(D)}$ \\ 1 Center for Biological Systems Analysis ZBSA, Albert-Ludwigs-University Freiburg, \\ 79104 Freiburg, Germany; lukas.braun@uniklinik-freiburg.de (L.M.B.); \\ simon.lagies@zbsa.uni-freiburg.de (S.L.) \\ 2 Department of General and Visceral Surgery, University of Freiburg, Medical Center Faculty of Medicine, \\ 79106 Freiburg, Germany \\ 3 Institute of Biology II, Albert-Ludwigs-University Freiburg, 79104 Freiburg, Germany \\ 4 Spemann Graduate School of Biology and Medicine, Albert-Ludwigs-University Freiburg, \\ 79104 Freiburg, Germany \\ 5 Department of Medicine I (Hematology, Oncology and Stem Cell Transplantation), University of Freiburg \\ Medical Center, 79104 Freiburg, Germany; rhena.klar@uniklinik-freiburg.de (R.F.U.K.); \\ saskia.hussung@uniklinik-freiburg.de (S.H.); ralph.fritsch@usz.ch (R.M.F.) \\ 6 SFB/Collaborative Research Center 850 (CRC 850)—Control of Cell Motility in Morphogenesis, \\ Cancer Invasion and Metastasis, University of Freiburg, 79104 Freiburg, Germany \\ 7 German Cancer Consortium, 79104 Freiburg, Germany \\ 8 Comprehensive Cancer Center Freiburg, 79104 Freiburg, Germany \\ 9 Department of Medical Oncology and Hematology, University Hospital of Zurich, 8091 Zurich, Switzerland \\ 10 BIOSS Centre for Biological Signalling Studies, University of Freiburg, 79104 Freiburg, Germany \\ * Correspondence: bernd.kammerer@zbsa.uni-freiburg.de (B.K.); uwe.wittel@uniklinik-freiburg.de (U.A.W.) \\ + These authors contributed equally to this paper.
}

Received: 11 May 2020; Accepted: 29 May 2020; Published: 1 June 2020

check for updates

\begin{abstract}
Pancreatic ductal adenocarcinoma (PDAC) is associated with high mortality and will become the second most common cause of cancer-associated mortality by 2030. The poor prognosis arises from a lack of sensitive biomarkers, limited therapeutic options, and the astonishingly high recurrence rate after surgery of $60-80 \%$. The factors driving this recurrence, however, remain enigmatic. Therefore, we generated patient-derived organoids (PDOs) from early- and late-recurrent PDAC patients. Cellular identity of PDOs was confirmed by qPCR, ddPCR, and IHC analyses. This is the first study investigating the metabolism in PDOs of different, clinically significant PDAC entities by untargeted GC/MS profiling. Partial least square discriminant analysis unveiled global alterations between the two sample groups. We identified nine metabolites to be increased in early recurrent PDOs in comparison to late recurrent PDOs. More than four-times increased were fumarate, malate, glutamate, aspartate, and glutamine. Hence, $\alpha$-keto acids were elevated in PDO-conditioned medium derived from early recurrent patients. We therefore speculate that an increased anaplerotic metabolism fuels the Krebs-cycle and a corresponding higher accessibility to energy fastens the recurrence in PDAC patients. Therein, a therapeutic intervention could delay PDAC recurrence and prolong survival of affected patients or could serve as biomarker to predict recurrence in the future.
\end{abstract}

Keywords: pancreatic ductal adenocarcinoma; PDAC; recurrence; relapse; metabolomics; GC/MS; patient derived organoids; PDO culture; anaplerotic TCA-cycle; glutamate 


\section{Introduction}

Pancreatic ductal adenocarcinoma (PDAC) is the most common histological subtype of pancreatic cancer and a highly aggressive and fast-growing type of cancer [1-3]. In about $90 \%$ to $95 \%$ of all pancreatic tumors, the cell-of-origin can be found in the pancreas ductal epithelium [1]. PDAC is associated with a very poor prognosis [4], is one of the major causes for cancer-related death in the western world [5] and is predicted to become the second-most cause of cancer-associated mortality [6] by 2030 [7]. The 5-year survival rate of PDAC patients has changed only little during recent years [5] and is now around $10 \%$ for all stages [8,9]. Important risk factors for PDAC include genetic predisposition including germline mutations in BRCA2 [10], CDKN2A [11], and CFTR [12] as well as smoking, diabetes, obesity and chronic pancreatitis $[4,13]$. The very low survival rates associated with PDAC are due to several factors, of which the most important are the very rapid progression, early microdissemination [14], and the late onset of any specific symptoms [15]. The majority of PDACs are diagnosed at an advanced, locally irresectable or metastatic stage when only palliative therapies can be offered [16]. Biologically, pancreatic cancers are locally highly infiltrative tumors with vascular and perineural invasion being observed frequently. Moreover, locoregional lymphatic and/or distant metastases are found at first diagnoses in the majority of cases [17]. The molecular pathology of PDAC is extremely challenging with a 92-95\% prevalence in non-druggable activating KRAS mutation next to TP53 and/or CDKN2A alterations driving cancer survival, growth, resistance to therapeutic options and metastasis [18]. These factors further contribute to a rapid progression of the disease [19] and support anabolic metabolic pathways [15]. About $80 \%$ of all patients suffering from pancreatic cancer have unresectable carcinoma at diagnosis, decreasing their estimated overall survival to only a few months [20]. The therapeutic options for these patients are limited to combination chemotherapy, which is associated with significant toxicity [15]. Many patients who undergo curative resection experience disease recurrence within one year after surgery [21-24].

For patients initially presenting with surgically resectable PDAC (approximately 20\%) [25], disease recurrence after initial curative surgery is the major challenge and impediment to long-term survival. Among all patients undergoing resection, cancer recurrence develops in up to $80 \%$ within time frames of clinical trials and are probably even higher in real-life populations [26-28]. Many studies found that the early post-operative recurrence is due to local and distant metastases [29]. Patients who developed distant metastases early after surgery have only a limited median survival time of a few months $[30,31]$. Studies trying to identify risk factors for recurrence after PDAC resection found tumor size, carbohydrate antigen 19-9 (CA19-9), perineural invasion, and lymph node metastases to correlate with disease recurrence [32-34]. However, it is likely that microscopic metastases have formed in the majority of patients prior to surgery or radiation therapy [15]. However, the mechanisms determining early or late PDAC recurrence after surgery have not yet been extensively studied [29-31] and better predictive biomarkers are required for more successful clinical stratification of patients.

During the last decades, many attempts in pancreatic cancer research focused on understanding molecular mechanisms within the tumor cells, the development of pancreatic cancer, and finding new therapeutic options to overcome treatment resistance [35]. Moreover, different studies tried to find novel biomarkers for the early detection of pancreatic cancer [36-38]. However, the use of the limited number of established pancreatic cancer cell lines in these studies was associated with several limitations. First, many cell lines were established from metastatic sites and do underrepresent characteristics found in primary tumors or pancreatic intraepithelial neoplasia (PanIN) lesions [39], and second, established cell lines grow extremely fast which could easily lead to the selection of different clones and a genetic drift as reported previously [40]. Application of 3D-grown conventional cell lines can in general overcome some issues of abnormal proliferation behavior observed in 2D-cultures in comparison to in situ situations [41]. Other research systems include patient-derived xenografts (PDX). However, these systems require large amounts of tissue [42] and need several months to be established [43]. Genetically engineered mouse models of PDAC could give good insights into the disease, but the systems are time-consuming, need a lot of space, and require high costs [44]. In order 
to overcome at least some of the limitations, 3D patient-derived pancreatic ductal organoids (PDO) can be cultured directly from primary PDAC tissue, pancreatitis tissue, or even healthy pancreas [45]. Organoids are a cellular 3D system with self-renewal and self-organization capacity which maintains an appearance and functionality comparable to their original tissue [39]. Moreover, the systems were found to be genetically stable throughout many passages $[46,47]$. Human PDAC organoids could generate PanIN lesions and develop into invasive PDAC after transplantation into mice [45]. With PDO cultures, PDAC of a variety of patients can be modeled in vitro and experimental findings can be correlated to the clinical course of the donating patients [45]. The organoid culture system allows the expansion of primary tissue in an in vitro system which is much closer to the actual patient than established cell lines are. Moreover, these systems allow the growth of 3D structures from healthy and disease tissue making the direct comparison between both states more convenient [48]. One of the major current limitations with organoid cultures from patient tissue is the limitation in cell expansion and the heterogenic growth [48]. The cultures need relatively long to grow and to deliver sufficient cell mass, compared to 2D cultures of established cell lines. Moreover, the organoid cultures depend on many supplemental factors to grow in vitro [39].

In this study, we generated different PDOs from early and late recurrent PDAC patients. After the evaluation of PDOs in regard to tissue-specificity, differences in primary metabolism between early and late recurrence should be unveiled by untargeted GC/MS profiling. We found that PDOs derived from early recurrent PDAC patients had higher levels of tricarboxylic acid intermediates and some anaplerotic amino acids, suggesting a higher capacity to generate energy. Thus, early recurrence might be the consequence of higher energy levels in these malignancies, suggesting a possible target to delay recurrence in future PDAC therapy or potential biomarkers to predict recurrence.

\section{Material and Methods}

\subsection{Preparation of Human Pancreas Organoid Feeding Medium}

Feeding medium for human pancreas organoid cultures (PDO) was prepared freshly at least every two weeks. The organoid splitting medium was prepared by supplementing Advanced DMEM/F-12 medium with $10 \mathrm{mM}$ HEPES and $1 \times$ GlutaMAX ${ }^{\mathrm{TM}}$. The ROCK inhibitor Y-27632 $(10.5 \mu \mathrm{M})$ was added to the feeding medium directly before use. For organoid feeding medium, following ingredients were added to the splitting medium (final concentrations are given): $100 \mu \mathrm{g} / \mathrm{mL}$ primocin, $500 \mathrm{nM}$ A38-01, $50 \mathrm{ng} / \mathrm{mL}$ mEGF, $100 \mathrm{ng} / \mathrm{mL}$ hFGF10, $10 \mathrm{nM}$ gastrin I, $1.25 \mathrm{mM}$ N-acetylcysteine, $10 \mathrm{mM}$ nicotinamide, $1 \times$ B27 supplement, 1× R-Spondin I-conditioned medium, 1× Wnt3a-conditioned medium and $10.5 \mu \mathrm{M}$ Y-27632.

\subsection{Cultivation and Passaging of Human Pancreas Organoid Cultures}

PDAC organoids were established [49] and cultured as described previously [45]. Organoids were grown in $25 \mu \mathrm{L}$ Matrigel Matrix Basement Membrane domes in 24-well plates. Cultures were passaged once a week and split 1:2 if well-growing. Feeding medium was replaced twice a week. For organoid passaging, matrigel domes were transferred into $10 \mathrm{~mL}$ cold splitting medium and kept on ice until centrifugation. All centrifugation steps were performed at $1200 \mathrm{rpm}$ and $4{ }^{\circ} \mathrm{C}$ for $5 \mathrm{~min}$. Organoids were centrifuged, splitting medium was discarded, cells were resuspended in $1 \mathrm{~mL}$ TrypLE Express (RT), and incubated at $35^{\circ} \mathrm{C}$ and $180 \mathrm{rpm}$ for $15 \mathrm{~min}$. Following, $9 \mathrm{~mL}$ of cold splitting medium were added into each tube and cells were centrifuged again. Organoid cell pellets were subsequently resuspended in cold Matrigel and immediately seeded into 24-well plates with $25 \mu \mathrm{L}$ Matrigel for each dome. Organoids were incubated at $37^{\circ} \mathrm{C}$ for $15 \mathrm{~min}$ before $500 \mu \mathrm{L}$ feeding medium were carefully added into each well. Organoids were cultured at $37^{\circ} \mathrm{C}$ and $5 \% \mathrm{CO}_{2}$ in a humidified atmosphere. 


\subsection{Isolation of Total RNA from Organoid Cultures}

For isolation of RNA from organoid cultures, matrigel domes were resuspended in $1 \mathrm{~mL}$ ice-cold $1 \times$ PBS. The organoids were centrifuged at $5500 \mathrm{rpm}$ and $4{ }^{\circ} \mathrm{C}$ for $6 \mathrm{~min}$. The organoid pellets were resuspended in $500 \mathrm{~mL}$ Cell Recovery Solution and incubated on ice for $60 \mathrm{~min}$. Following, the organoids were centrifuged (same settings as before) and washed once with $500 \mu \mathrm{L}$ ice-cold $1 \times$ PBS. The supernatant was discarded and the organoid pellets were immediately frozen at $-80{ }^{\circ} \mathrm{C}$. RNA was isolated using the RNeasy ${ }^{\circledR}$ Plus Mini Kit (Qiagen, Hilden, Germany) and QIAshredder (Qiagen) according to manufacturer's instructions. Briefly, organoid pellets were resuspended in $350 \mu \mathrm{L}$ Buffer RLT Plus, loaded on a QIAshredder column and centrifuged at 13,000 rpm for $2 \mathrm{~min}$. The homogenate was transferred to a gDNA Eliminator spin column in a new collection tube and centrifuged for $30 \mathrm{~s}$ at 10,000 rpm. A total volume of $525 \mu \mathrm{L} \mathrm{100 \%} \mathrm{ethanol} \mathrm{was} \mathrm{added} \mathrm{to} \mathrm{the} \mathrm{flow-through} \mathrm{and} \mathrm{shortly}$ vortexed. $700 \mu \mathrm{L}$ of the mixture were loaded onto an RNeasy Mini spin column and centrifuged at 10,000 rpm for $30 \mathrm{~s}$. The flow-through was discarded and the step was repeated until the whole sample passed the membrane. Following, $500 \mu \mathrm{L}$ Buffer RPE was added to the spin column and centrifuged at 10,000 rpm for $30 \mathrm{~s}$. The flow-through was discarded and the step was repeated once. The mini spin column was centrifuged at 13,000 rpm for $2 \mathrm{~min}$ to dry the membrane. The column was placed in a new collection tube, $35 \mu \mathrm{L}$ RNase-free water were added onto the membrane and columns were incubated at RT for $3 \mathrm{~min}$. Following, samples were centrifuged at 13,000 rpm for $2 \mathrm{~min}$ to elute total RNA.

\subsection{Synthesis of cDNA from RNA Templates}

The cDNA from RNA templates was synthesized using the RevertAid First Strand cDNA Synthesis kit (Thermo Scientific, Waltham, MA, USA). Briefly, $1 \mu \mathrm{g}$ total RNA was incubated with $0.5 \mu \mathrm{L}$ OligodT and $0.5 \mu \mathrm{g}$ Random Hexamer primer for $5 \mathrm{~min}$ at $70{ }^{\circ} \mathrm{C}$ in a total volume of $12 \mu \mathrm{L}$. Following, $4 \mu \mathrm{L}$ Reaction Buffer (5x), $2 \mu \mathrm{L}$ dNTP mix and $1 \mu \mathrm{L}$ RNase inhibitor were added and the mix was incubated for $5 \mathrm{~min}$ at $37^{\circ} \mathrm{C}$. After addition of $1 \mu \mathrm{L}$ RevertAid transcriptase, the reaction was run for $10 \mathrm{~min}$ at $25{ }^{\circ} \mathrm{C}, 60 \mathrm{~min}$ at $42{ }^{\circ} \mathrm{C}$ and $10 \mathrm{~min}$ at $70{ }^{\circ} \mathrm{C}$. The cDNA was diluted to $3 \mathrm{ng} / \mu \mathrm{L}$ for qPCR analysis. Additionally, total RNA was diluted to $3 \mathrm{ng} / \mu \mathrm{L}$ for noRT-control.

\subsection{Design of Primer Sequences for $q P C R$}

Primers were designed for use with SYBR Green in qPCR assays. The mRNA sequences of the respective genes were downloaded from $\mathrm{NCBI} /$ gene website and primers were designed using the primer-BLAST tool (NCBI, Bethesda, MD, USA). All primers were designed with a GC content from $40 \%$ to $60 \%$, a melting temperature between $58^{\circ} \mathrm{C}$ and $60^{\circ} \mathrm{C}$ with an optimum at $60^{\circ} \mathrm{C}$ and a primer length between $18 \mathrm{bp}$ and $22 \mathrm{bp}$ with an optimum at $20 \mathrm{bp}$. Sequences are shown in Table S1.

\subsection{Quantitative PCR ( $q P C R$ ) Analysis}

For analysis of relative gene expression of epithelial and fibroblast markers in PDOs and cell lines, primers were diluted to $7.5 \mu \mathrm{M}$ in $\mathrm{H}_{2} \mathrm{O}$. A total amount of $9 \mathrm{ng}$ cDNA or noRT-control was loaded together with $0.5 \mu \mathrm{L}$ fwd-primer, $0.5 \mu \mathrm{L}$ rev-primer and $5 \mu \mathrm{L}$ Power SYBR Green PCR Master Mix (Thermo Scientific) in a final volume of $10 \mu \mathrm{L}$ in triplicates in 384-well qPCR plates. Negative controls lacking cDNA were included in the assay. The qPCR was run with the LightCycler 480 Instrument II (Roche, Basel, Switzerland) using the following program: $10 \mathrm{~min}$ at $95^{\circ} \mathrm{C}$, followed by 40 cycles denature for $15 \mathrm{~s}$ at $95^{\circ} \mathrm{C}$ and annealing for $60 \mathrm{~s}$ at $60^{\circ} \mathrm{C}$. The relative expression of target genes was normalized to $\beta$-actin expression and calculated as fold-change over all samples using the $2^{-\Delta \Delta C t}$ method.

\subsection{Isolation of DNA from Organoid Cultures}

For DNA isolation from the organoid cultures, matrigel domes were each resuspended with $1 \mathrm{~mL}$ ice-cold $1 \times$ PBS and transferred to an Eppendorf tube. The organoids were then centrifuged at $5300 \mathrm{rpm}$ 
and $4{ }^{\circ} \mathrm{C}$ for $5 \mathrm{~min}$. The supernatant was discarded and the organoid pellet was resuspended in $500 \mu \mathrm{L}$ Cell Recovery Solution and incubated on ice for one hour. Organoids were then centrifuged again at $5300 \mathrm{rpm}$ and $4^{\circ} \mathrm{C}$ for $5 \mathrm{~min}$ and the supernatant was removed. The pellet was washed with $500 \mu \mathrm{L}$ ice-cold $1 \times$ PBS and centrifuged for $10 \mathrm{~min}$ at $5300 \mathrm{rpm}$ and $4{ }^{\circ} \mathrm{C}$. The supernatant was discarded and the organoid pellet was frozen at $-80{ }^{\circ} \mathrm{C}$ until DNA isolation. DNA was isolated with the QIAamp DNA Micro Kit (Qiagen) using the protocol "Isolation of Genomic DNA from Tissues" according to the manufacturer's instructions. DNA was frozen at $-20^{\circ} \mathrm{C}$ until subsequent ddPCR analyses.

\subsection{Droplet Digital PCR (ddPCR)}

Locked nucleic acid (LNA) probes and corresponding primer pairs for KRAS mutations were designed using Beacon Designer v.8.20 software (Premier Biosoft, Palo Alto, CA, USA). Primers and probes were manufactured by Integrated DNA Technologies (IDT, Inc., Coralville, IA, USA). Detailed information on primer and probe design as well as the corresponding sequences have been published previously [50]. Primers, probes, $2 \mu \mathrm{L} /$ well template DNA, and nuclease-free water (Ambion) were added to ddPCR Supermix for Probes (Bio-Rad, cat. no \#186-3024). Reaction mix was set up as recommended and droplets were generated using a QX100/200TM Droplet Generator (Bio-Rad, cat. no. \#1863002) following manufacturer's instructions. Each sample was assayed in quadruplicates. Following Droplet Generation, droplets were transferred into a 96-well PCR plate (Bio-Rad, cat. no. \#12001925). PCR was run on a C1000 TouchTM Thermal Cycler (Bio-Rad, cat. no. \#1851197), and samples were subsequently analyzed on a QX100/200TM Droplet Reader (Bio-Rad, cat. no. 1863003) using QuantaSoft v1.7.4.0917 (Bio-Rad, cat.no. \#1864011). PCR protocols for the corresponding KRAS assays, the respective assay controls, and data analysis were performed as described previously [50].

\subsection{Embedding Organoids for Immunohistochemistry (IHC) Analysis}

For IHC analysis, organoid domes were fixed in $500 \mu \mathrm{L} 4 \%$ paraformaldehyde (PFA) solution at RT for 15 min after removal of feeding medium. PFA was removed and domes were embedded in $1 \mathrm{~mL}$ of $2 \%$ agarose $(w / v$; in water) solution. After polymerization, the embedded domes were stored in $50 \%$ ethanol $(v / v)$ at $4{ }^{\circ} \mathrm{C}$ until further processing. The domes were embedded in paraffin, cut into $3 \mu \mathrm{m}$ sections and placed on coverslips.

\subsection{Immunohistochemistry Staining}

For IHC staining, sections were incubated overnight at $37^{\circ} \mathrm{C}$, deparaffinized and boiled in a pressure cooker in $10 \mathrm{mM}$ citrate buffer ( $\mathrm{pH} \mathrm{6.0,} \mathrm{supplemented} \mathrm{with} 0.05 \%$ Tween20) for $15 \mathrm{~min}$. Endogenous peroxidase activity was quenched with peroxidase-blocking-solution for $30 \mathrm{~min}$, followed by $3 \times 5$ min wash in TBS-T (pH 7.6, 0.05\% Tween 20 ). The background was blocked with $1 \%$ BSA/TBS for 30 min. Primary antibodies were diluted in 1\% BSA/TBS ( $\alpha$ SMA 1:400, CK19 1:400, PDX1 1:1000, Vimentin 1:400) and slides were incubated with the respective antibodies overnight at $4{ }^{\circ} \mathrm{C}$. Slides were washed $3 \times 5 \mathrm{~min}$ in TBS-T and incubated with EnVision+/HRP-labeled polymer anti-rabbit or anti-mouse for $60 \mathrm{~min}$ at RT. Slides were washed again $3 \times 5 \mathrm{~min}$ in TBS-T. Stainings were visualized by incubation with the Liquid DAB + Substrate Chromogen System (DAKO, 1:51) for 2 min, followed by washing in water. Counter stain was done with Mayer's hematoxylin (1:5, v/v, in water) for 5 min, followed by incubation in water $\left(37^{\circ} \mathrm{C}\right)$ for $10 \mathrm{~min}$. The coverslips were mounted using the Rotihistokitt II (Roth, Karlsruhe, Germany). Details of the antibodies used in this study are depicted in Table S2.

\subsection{Organoid Harvest and Metabolite Extraction}

Organoids were passaged as described previously and cultured for eight days until matrigel domes were confluent for harvesting. The plates were placed on ice, the medium was removed and the matrigel domes were disrupted in $1 \mathrm{~mL}$ ice-cold $0.9 \% \mathrm{NaCl}$ solution by thoroughly pipetting up and down and transferred into $15 \mathrm{~mL}$ canonical tubes. Organoids from three domes were pooled for one analysis sample and washed thrice with $10 \mathrm{~mL}$ ice-cold $0.9 \% \mathrm{NaCl}$ solution to remove the matrigel 
from the cells. The metabolism was quenched by the addition of $1.5 \mathrm{~mL}$ ice-cold extraction buffer ( $\mathrm{MeOH} / \mathrm{H} 2 \mathrm{O}$ 9:1 (v/v), $1 \mu \mathrm{g} / \mathrm{mL}$ ribitol, $1 \mu \mathrm{g} / \mathrm{mL}$ phenyl- $\beta$-D-glucopyranoside) and the suspension was transferred into $2 \mathrm{~mL}$ screw-cap-tubes which had been pre-filled with $300 \mathrm{mg}$ glass beads. The cells were snap-frozen in liquid nitrogen and stored at $-80^{\circ} \mathrm{C}$ until further processing. The cell samples were applied to a Precellys Evolution tissue homogenizer for cell lysis with the following settings: $3 \times 15 \mathrm{~s}$ at $6500 \mathrm{rpm}$ with a $10 \mathrm{~s}$ break between the homogenization steps. The samples were subsequently centrifuged at $20,000 \mathrm{rcf}$ and $4{ }^{\circ} \mathrm{C}$ for $10 \mathrm{~min}$ to separate cell debris. $1200 \mu \mathrm{L}$ of the supernatant were dried under vacuum and analyzed by GC/MS.

\subsection{Metabolite Extraction from PDO Conditioned Medium}

Proteins were precipitated and metabolites extracted by cold acetonitrile:methanol as described in Lagies et al. [51]. Vacuum dried metabolite pellets were subjected to GC/MS analysis.

\subsection{GC/MS Based Metabolic Profiling}

Dried metabolite pellets were subjected to untargeted gas chromatography coupled to mass spectrometry (GC/MS) based profiling as described previously [51]. In summary, metabolites were derivatized by methoxyamine hydrochloride in pyridine followed by silylation with $N$-trimethylsilyl- $N$ methyl trifluoroacetamide. Samples were splitlessly injected onto an HP-5MS column $(60 \mathrm{~m} \times 0.25 \mathrm{~mm}$ $\times 0.25 \mu \mathrm{m})$ in randomized order with regular quality control samples in between. Metabolites were analyzed by electron ionization and annotated according to retention index and mass spectral similarity to different libraries, including an in-house database. Features were aligned by SpectConnect and normalized to internal standard and peak-sum. Statistical analysis was conducted with MetaboAnalyst 4.0.

\subsection{Statistical Analysis}

For others than untargeted metabolomics profiling data, statistics were performed using the GraphPad prism software. Normally distributed data were compared using an unpaired $t$-test. Data are displayed as mean \pm standard error of the mean. A $p$-value $<0.05$ was considered as statistically significant. Significances were shown with symbols $\left({ }^{*}: p<0.05,{ }^{* *}: p<0.01,{ }^{* * *}: p<0.001\right)$.

\subsection{Data Avialbility}

Results and statistical results of metabolic profiling are provided in supplementary data files Tables S3 and S4 for endometabolites and exometabolites, respectively. Other data will be available upon reasonable request.

\subsection{Compliance with Ethical Standards}

Informed consent was obtained for the development and usage of 3D Organoid cultures from human pancreatic cancer tissue and approved by the local Ethics Committee of the Albert-LudwigsUniversity Freiburg, Freiburg, Germany (126/17; 28 March 2017).

\section{Results}

\subsection{Patient and Clinical Data of Organoid Lines Used in This Study}

Patient-specific data of the patient-derived organoid lines used in this study are summarized in Table 1. The table contains information about patient sex and year of birth, additional to clinical information about the tumor grading, the KRAS mutation, and the recurrence state of the tumor. "Early recurrence" summarizes tumor recurrence within the first six months after curative resection, whereas "late recurrence" indicates disease recurrence more than six months after surgery. 
Table 1. Patient and clinical data of PDO lines used in this study. The table summarizes early recurrent (ER) and late recurrent (LR) patients data including sex and age, as well as clinical data including tumor grading, KRAS mutation and state of tumor recurrence. Patient ER-1 received three cycles FOLFIRINOX prior to resection, while all other patients did not receive any neoadjuvant chemotherapy.

\begin{tabular}{|c|c|c|c|c|c|c|c|c|c|c|}
\hline $\begin{array}{l}\text { Organoid } \\
\text { Line }\end{array}$ & Sex & $\begin{array}{l}\text { Age/ } \\
\text { Years }\end{array}$ & TNM & CRM & $\begin{array}{l}\text { Tumor } \\
\text { Size/cm }\end{array}$ & Tumor Location & $\begin{array}{l}\text { PDAC } \\
\text { Grading }\end{array}$ & $\begin{array}{l}\text { KRAS } \\
\text { Mutation }\end{array}$ & $\begin{array}{c}\text { Time to, } \\
\text { Location of } \\
\text { Recurrence/days }\end{array}$ & $\begin{array}{c}\text { Survival (Last } \\
\text { Follow Up) after } \\
\text { Resection/days }\end{array}$ \\
\hline ER-1 & Male & 32 & pT3, pN1 (3/14). L0. V0. Pn1 & R1 & 6.5 & Pancreas head & G2 & G12R & 101, Liver & $(1172)$ \\
\hline ER-2 & Male & 67 & pT3, pN1 (7/27). L1. V0. Pn1 & $\mathrm{R} 1$ & 4.5 & Pancreas head & $\mathrm{G} 2$ & G12D & 69, Liver & $(1106)$ \\
\hline ER-3 & Female & 66 & pT3, pN2 (5/37). L1. V1. Pn1 & Narrow & 5.0 & Pancreas body & $\mathrm{G} 2$ & G12D & 42, Liver & 94 \\
\hline LR-1 & Male & 77 & pT3, pN2 (6/16). L1. V0. Pn1 & Narrow & 5.0 & Pancreas head & G3 & G12R & 538, Lung & 886 \\
\hline LR-2 & Male & 72 & pT2, pN2 (4/22). L1. V0. Pn1 & Narrow & 3.8 & Pancreas head & G3 & G12D & 431 & $(431)$ \\
\hline LR-3 & Male & 58 & pT2, pN1 (1/19). L1. V0. Pn0 & $\mathrm{R} 1$ & 3.2 & Pancreas head & G2 & G12D & 492, Liver local & (543) \\
\hline
\end{tabular}




\subsection{Analysis of Pancreas and Fibroblast Lineage Marker Expression}

In order to control for the presence of pancreatic ductal cells versus fibroblasts in organoid cultures, the expression of lineage markers for both cell types was analyzed by qPCR (Figure 1). Gene expression was analyzed in PDO cultures, in myofibroblast-like pancreatic stellate cells (PSC, EP1077) and the established PDAC cell lines Capan-2, HPAF-II, MiaPaCa-2, and PANC-1 (PDAC). Expression is shown as fold-change over all samples. Most of the organoid lines expressed the ductal lineage markers cytokeratin 19 (KRT19) and SRY-Box transcription factor 9 (Sox9). Expression in PDAC cell lines was lower. RNA derived from PSC was used as a negative control for these markers. The results indicated a high content of ductal adenocarcinoma cells in the organoid cultures. Additionally, the expression of alpha smooth muscle actin (ACTA2) and vimentin (VIM) was analyzed as markers for mesenchymal cells and fibroblasts in the cultures. ACTA2, a marker for activated pancreatic stellate cells and cancer-associated fibroblasts, was detected in all organoid lines at very low levels. A high expression was found in PSC, serving as a positive control for fibroblast markers. Additionally, vimentin expression was low in all organoid lines, except for the chronic pancreatitis and the pseudocyst samples. Vimentin is a typical marker for mesenchymal-like cells. The high expression of vimentin in PDAC cell lines confirmed their mesenchymal type described previously [52].

Next, we assessed the mutation allele frequencies (MAFs) of the KRAS-mutations by ddPCR. All PDO harbored high fractions of the corresponding KRAS-mutation (Figure S1). Hence, these fractions were starkly elevated in comparison to the primary biopsies, indicating a very pure tumor population.

Besides gene expression analysis, lineage markers were also analyzed by immunohistochemistry staining. PDO sections were stained with antibodies against cytokeratin 19 (CK19) and pancreatic and duodenal homeobox 1 (PDX1) as epithelial and pancreas duct lineage markers (Figure 2, left panels), as well as with antibodies against alpha smooth muscle actin ( $\alpha \mathrm{SMA})$ and vimentin as fibroblast and mesenchymal lineage markers (Figure 2, right panels). The images are grouped according to the clinical group used for analysis. Pancreas tissue served as control. CK19 was found at high levels in all samples. Levels of PDX1 were much more variable with low and high expressing lines in all groups. ER-1 (early recurrent) and LR-1 (late recurrent) displayed lower levels of PDX1 compared to all other lines. Staining against $\alpha$ SMA and vimentin revealed a low level or even negative staining in all PDOs. The findings are in line with qPCR results. In conclusion, all organoid lines contained high amounts of epithelial and ductal cells, confirming them as a suitable system to study pancreatic ductal adenocarcinomas in vitro. However, also fibroblast markers were found, representing either some fibroblasts in PDO cultures or an EMT phenotype, as reported for PDAC cell lines [52]. 
a)

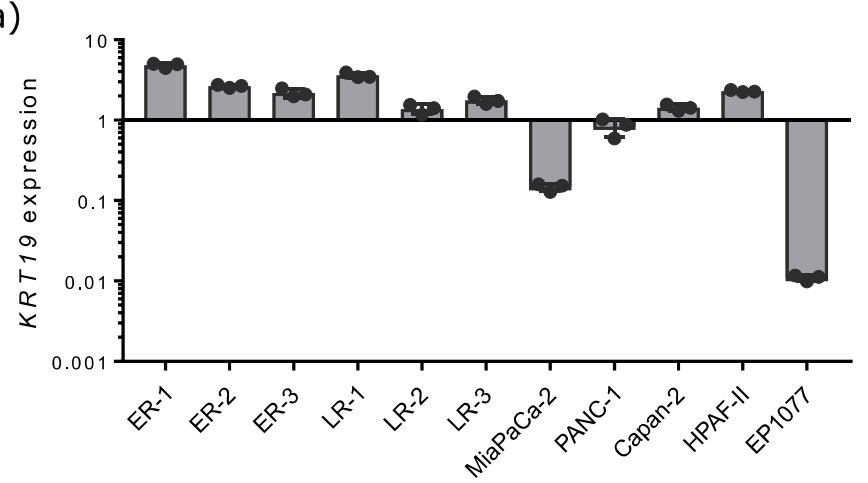

b)

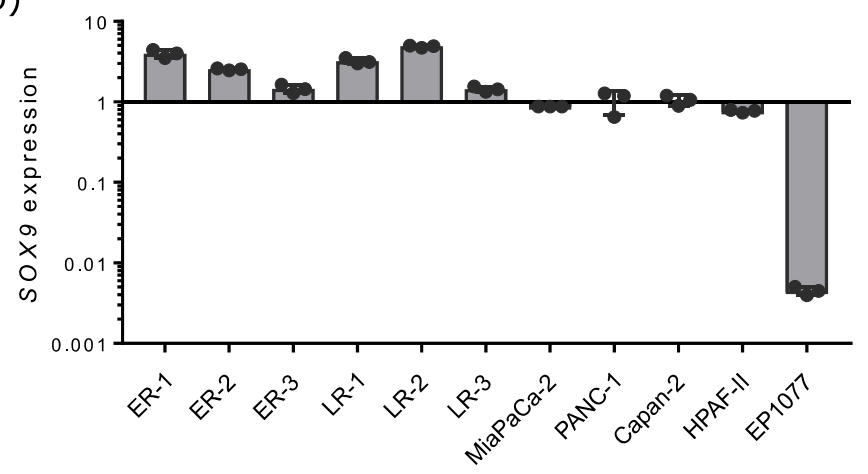

c)

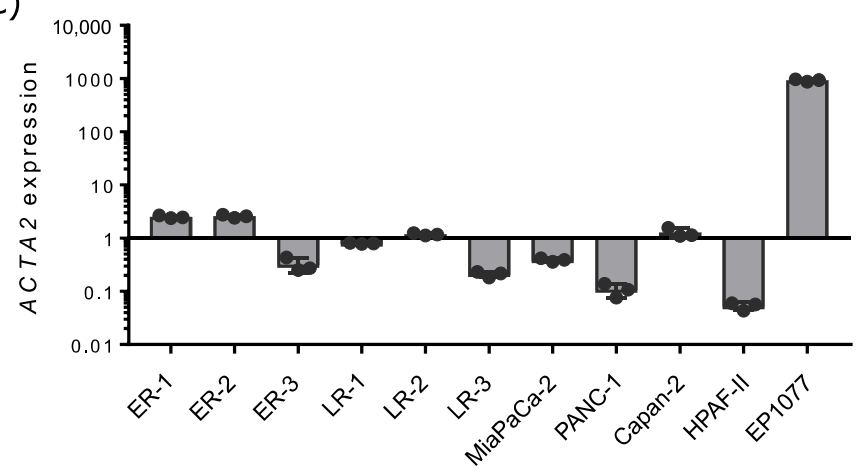

d)

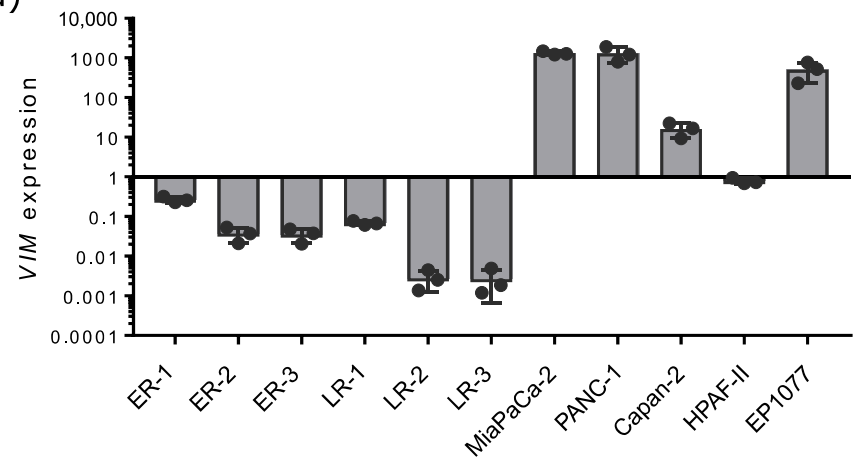

Figure 1. Gene expression analysis of epithelial, ductal and mesenchymal markers in PDOs, PDAC cell lines and PSCs. The expression of cytokeratin 19 (KRT19; $\mathbf{a})$, SOX9 (b) alpha smooth muscle actin $(A C T A 2 ; \mathbf{c})$ and vimentin $(V I M ; \mathbf{d})$ was analyzed by qPCR and normalized to $\beta$-actin expression. Gene expression was analyzed in early and late recurrent PDOs as well as in a pancreatic stellate cell line (EP1077) and the established PDAC cell lines Capan-2, HPAF-II, MiaPaCa-2 and PANC-1. Results are depicted in a logarithmic scale as fold change over all samples. Bar charts display mean \pm standard error of the mean $(n=3)$. 


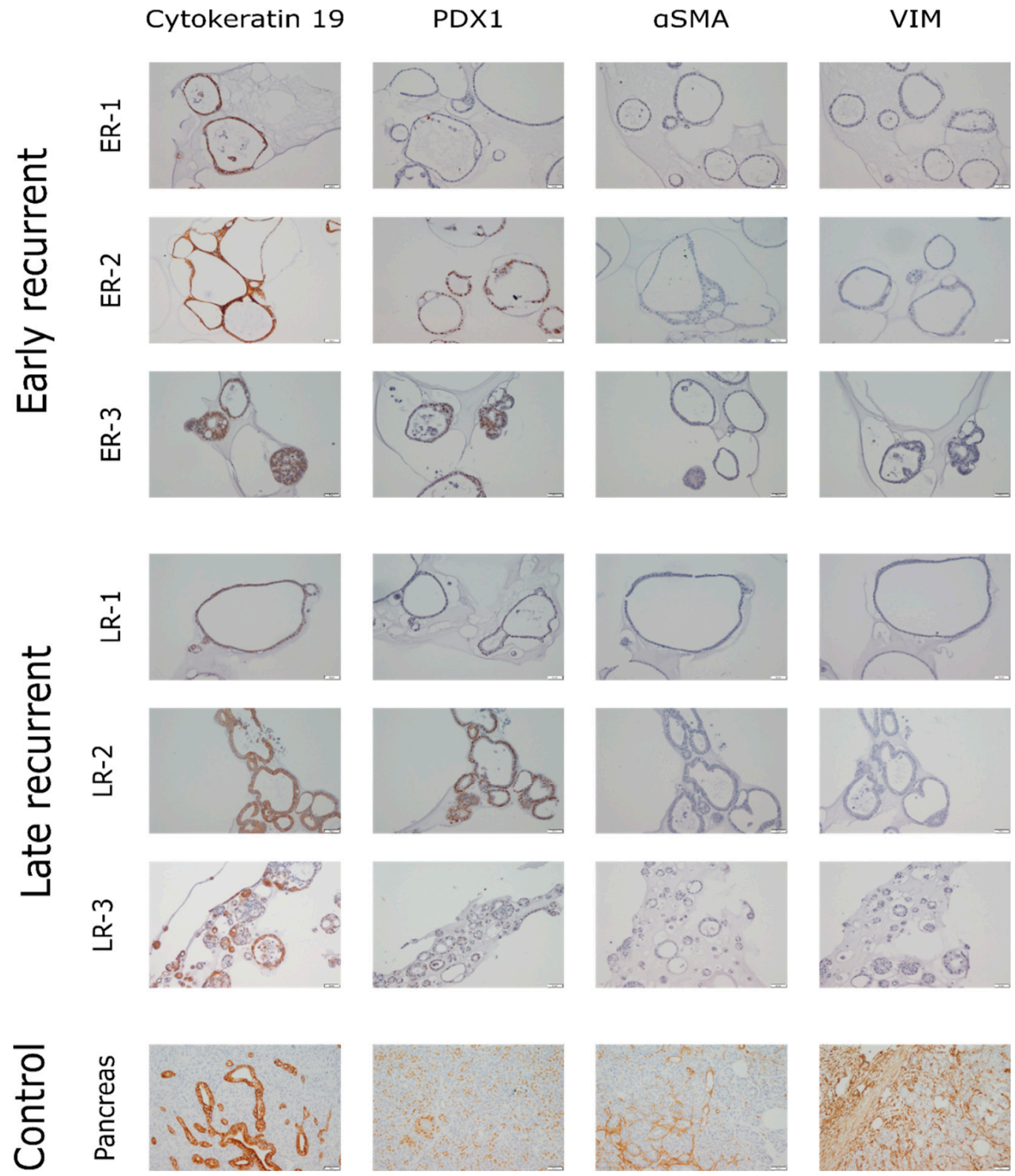

Figure 2. Immunohistochemistry staining of PDOs. Paraffin-embedded organoids were cut into $3 \mu \mathrm{m}$ sections and stained with antibodies against CK19 (first column) or PDX1 (second column) as markers for epithelial and ductal cells as well as antibodies against $\alpha \mathrm{SMA}$ (third column) or vimentin (fourth column) as markers for fibroblasts and mesenchymal cells. Brown color represents a positive signal. Pancreatic tissue served as control. Each panel depicts approximately $500 \times 675 \mu \mathrm{m}$.

\subsection{Metabolic Analysis of PDOs Derived from Early and Late Recurrent PDAC Patients}

Following validation of the organoid cultures, untargeted metabolomics analysis was conducted in early passage PDOs. For that, each PDO culture was measured in at least three technical replicates. As early and late recurrent PDAC patients could clearly be assigned to different clinical entities, we conducted a partial least square discriminant analysis (PLS-DA). Indeed, PLS-DA revealed that there were significant global alterations within the two metabolite pools, as shown by separated confidence intervals (see Figure 3a).

Next, we analyzed which metabolic pathways were differently regulated in early and late recurrent PDOs. Indeed, several pathways were affected when comparing early and late recurrent PDAC patients (see Figure 3b). Table 2 summarizes the pathway entities from Figure $3 \mathrm{~b}$ with corresponding pathway 
impact and statistical evaluation. More than half of the most affected pathways regulated differently in early and late recurrent PDOs were the TCA-cycle itself or anaplerotic pathways, closely connected to the TCA-cycle.

a)

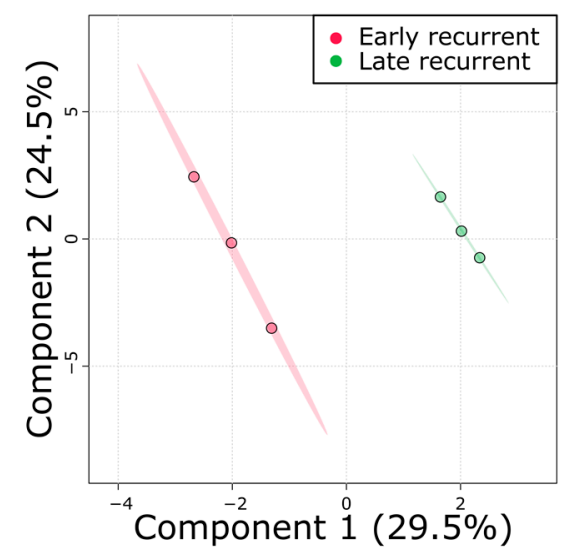

b)

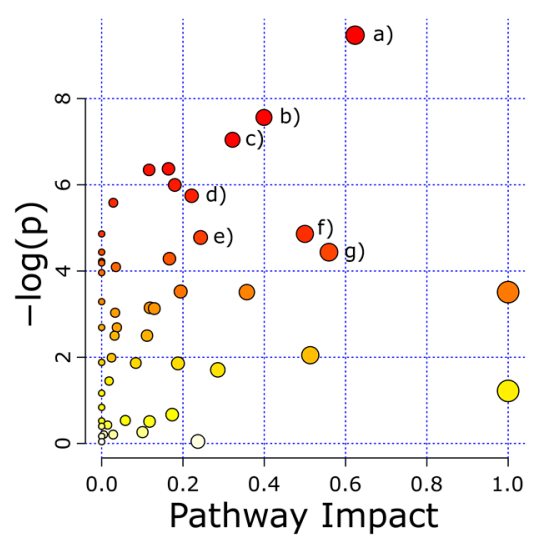

c)

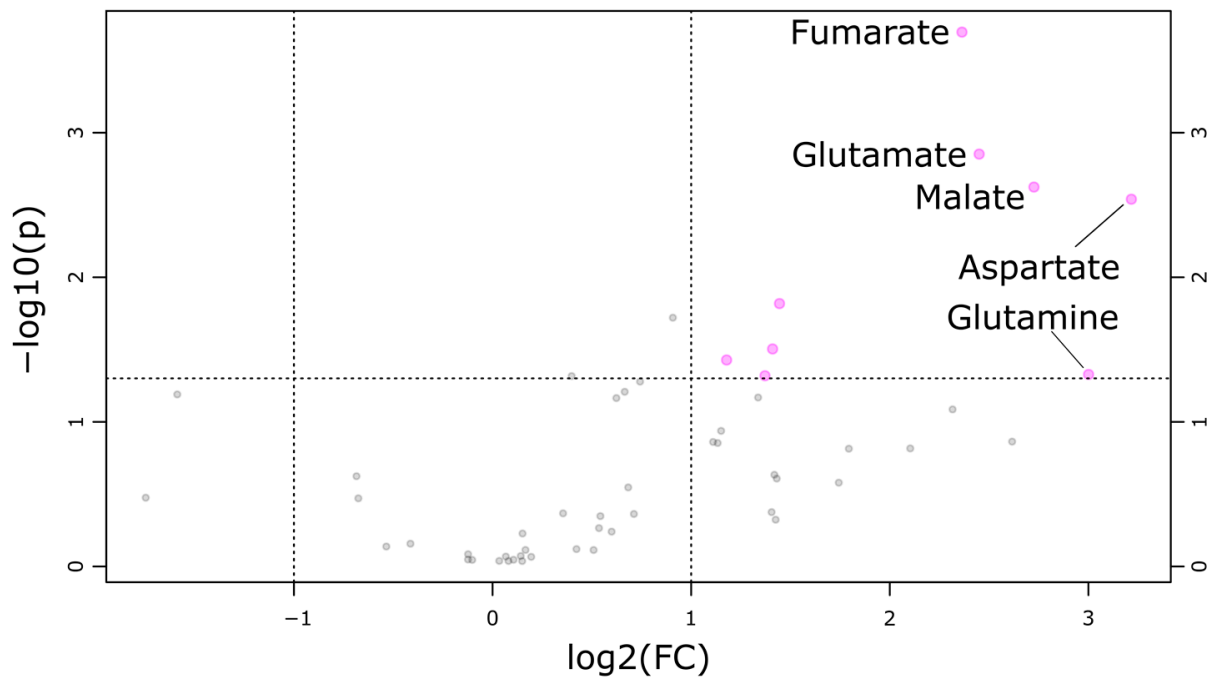

Figure 3. Metabolic profiling revealed tricarboxylic acid cycle (TCA-cycle) intermediates and anaplerotic amino acids were increased in early recurrent PDAC. (a) Partial least square discriminant analysis of PDOs derived from early (red) and late (green) recurrent PDAC patient unveiled global metabolic alterations. (b) Global pathway analysis revealed different pathways affected in PDOs. Identity of the pathways matching the cut-off values $(-\log (p)>4$ and pathway impact $>0.2)$ are depicted in table 2 . (c) Volcano plot analysis of identified metabolites. Early recurrent to late recurrent fold changes are displayed as $\log 2$ values. Only top five altered metabolites are labeled. Each data point represents at least three technical replicates of the different PDOs.

To further elucidate which metabolites contributed most to this separation, we conducted a volcano-plot analysis (Figure 3c). Fumarate and malate were the most increased TCA-cycle intermediates in PDOs derived from early recurrent PDAC patients. In parallel, glutamine, glutamate, and aspartate were also starkly elevated in the early recurrence group. These metabolites are in line with the identified pathways from Figure $3 b$ and Table 2.

In addition to the PDOs themselves, the PDO-conditioned culture medium was subjected to metabolic profiling. PLS-DA revealed again a discrimination between early and late recurrence 
(Figure 4a). The hypothesis of anaplerotic TCA-cycle activity in early recurrent PDAC could be substantiated by volcano plot analysis (Figure $4 \mathrm{~b}$ ) with the following observations: First, culture medium of PDOs derived from late recurrent PDAC patients had less glucose, which indicates that they relied more on glucose as an energy source. Second, asparagine was reduced in the medium of early recurrent PDOs, suggesting a higher import. Third, elevated secretion of $\alpha$-keto carboxylic acid, including branched-chain ketoacids (BCKAs), in the early recurrence group indicates a higher activity of transaminases. BCATs (BCKA amino transferases) use branched chain amino acids to produce glutamate and BCKAs and ALT (alanine amino transferase) uses alanine to form glutamate and pyruvate. Therefore, these results are also in line with the highly increased intracellular glutamate concentration in early recurrent PDOs.

\section{a)}

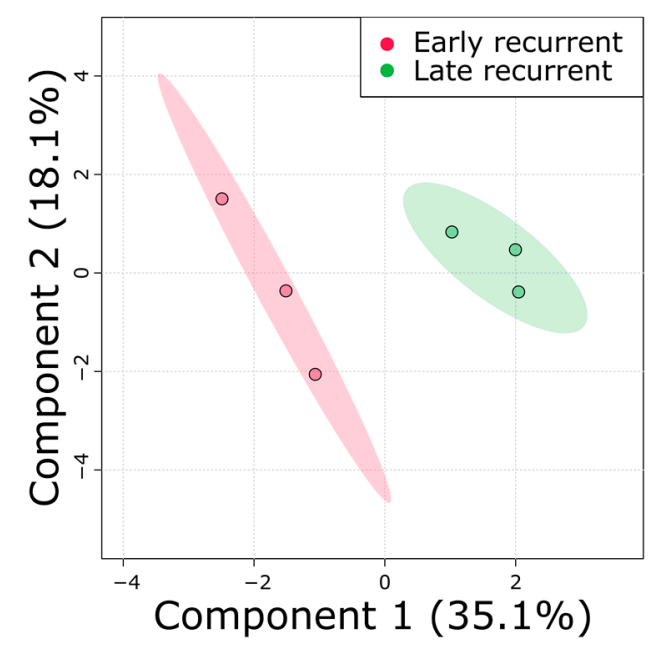

b)

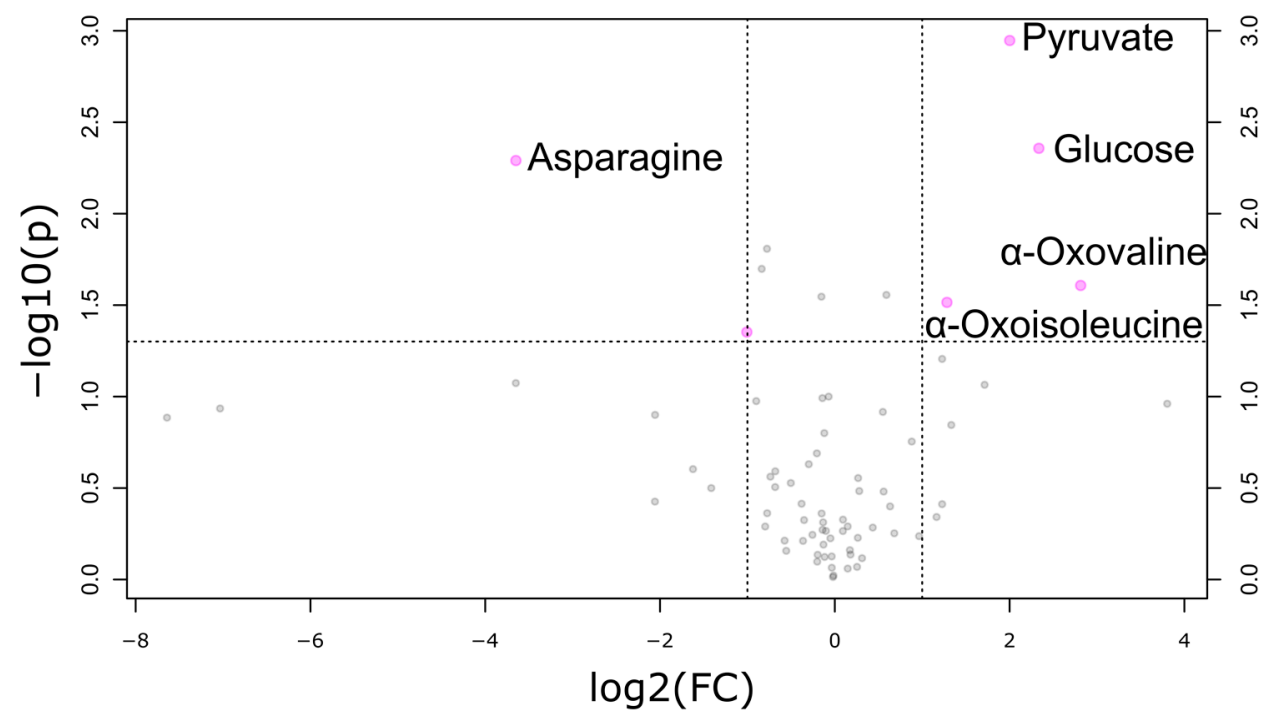

Figure 4. Exometabolome analysis of PDAC derived PDOs. (a) PLS-DA revealed discrimination between metabolites excreted from early recurrent PDOs (red) and late recurrent PDOs (green). (b) Volcano plot analysis of extracellular metabolites. Early recurrent to late recurrent fold changes are displayed as $\log 2$ values. Only top five metabolites are labeled. Each data point represents at least three technical replicates of the different PDOs. 
Table 2. Pathway entities with quality criteria and statistics from analysis shown in Figure $3 b$.

\begin{tabular}{cccc}
\hline Pathway Abbreviation & Pathway & Pathway Impact & $-\log (\mathbf{p})$ \\
\hline (a) & Alanine, aspartate and glutamate metabolism & 0.6234 & 9.4709 \\
(b) & beta-Alanine metabolism & 0.39925 & 7.5625 \\
(c) & Pyruvate metabolism & 0.32192 & 7.0445 \\
(d) & Histidine metabolism & 0.22131 & 5.7457 \\
(e) & TCA cycle & 0.24338 & 4.7773 \\
(f) & Glutamine and glutamate metabolism & 0.5 & 4.8607 \\
(g) & Starch and sucrose metabolism & 0.55921 & 4.4369 \\
\hline
\end{tabular}

\section{Discussion}

In this study, we successfully generated and validated patient-derived organoid cultures from PDAC patients, who went on to developed recurrence after surgical resection either within six months (early recurrence) or later than six months (late recurrence). We demonstrated tissue identity of these PDO cultures by both mRNA analysis and immunohistochemistry analysis of typical lineage markers. ddPCR unraveled high MAFs of KRAS in all PDOs, further validating our cultures as cancer organoids. To the best of our knowledge, this is the first study comparing metabolites in PDOs from these two distinct, clinically significant PDAC subgroups. Of note, organoids of all patients were generated from resected primary biopsies at the time of operation when further disease course was still obscure. We could uncover higher levels of anaplerotic amino acids and TCA-cycle intermediates in the early recurrence group. In addition, the analysis of exometabolites unveiled elevated levels of $\alpha$-keto acids and glucose together with decreased levels of asparagine in medium conditioned by early recurrent PDOs. Dufour and colleagues showed that PDAC bears in general low expression of asparagine synthetase and therefore is in high need for extracellular asparagine [53]. They suggested that an asparagine-low diet could have beneficial effects for PDAC patients. As we could show diminished levels of asparagine, we speculate that such an asparagine starvation could also delay PDAC recurrence after surgical resection.

Higher extracellular levels of glucose in early recurrent PDAC indicate a decreased dependency on glycolysis in comparison to late recurrent PDAC PDOs. Viale et al. have already proposed that a PDAC sub-population could be responsible for PDAC relapse and elegantly showed an increased reliance on the TCA-cycle within this population [54]. As a consequence, they proposed that targeting the TCA-cycle might be a potential treatment option for PDAC therapy. Therefore, we think that in agreement with our results (higher extracellular glucose and higher intracellular TCA-cycle intermediates) these metabolic alterations might not only serve as a clinically applicable biomarker of early versus late disease recurrence but also as a therapeutic target to delay recurrence after surgical therapy. Of note, we here present a pilot study with low sample numbers and larger prospective trials are needed to confirm our findings in a larger patient population.

The laboratory of Joshua Rabinowitz compared metabolite concentrations in pancreatic cancer tissue with adjacent benign tissue and uncovered relatively higher levels of glutamic acid in pancreatic malignant tissue [55]. However, the amino acids which were relatively lower were nitrogen donors. Therefore, they speculated that increased deamination led to the opposing abundance of specific amino acids. In another study, they showed that in almost all tissues the TCA-cycle is mainly fueled by lactate, with the exception of the pancreas, which uses primarily glutamine/glutamate for this purpose [56]. These results are again in line with our observation of increased consumption of nitrogen donors and increased intracellular glutamate levels in early PDAC PDOs, which can be seen as more malignant tissue in comparison to PDOs derived from late recurrent PDAC patients.

In humans, deamination reactions are normally catalyzed by transaminases, in which an amine group of an $\alpha$-amino acid is transferred, resulting in glutamic acid and an $\alpha$-keto carboxylic acid. Not only have we detected elevated levels of glutamic acid within the early recurrent PDO-cultures, but also have we detected several $\alpha$-keto carboxylic acids in those supernatants. Therefore, it is 
reasonable that higher transaminase activities also go along with an earlier relapse of PDAC. Confirmatory, Li et al. have proven high levels of mitochondrial branched-chain amino acid aminotransferase (BCAT2) in human PDAC. Subsequently, they showed in a Kras-driven PDAC mouse model that pancreas weight is significantly reduced in bcat2-/- PDAC mice [57]. Whereas BCAT2 is elevated in PDAC, enzymes metabolizing branched chained keto acids (BCKAs), i.e., BCKA-dehydrogenases are simultaneously decreased [58]. This further explains the elevated levels of BCKAs found in PDO-conditioned medium in the early recurrence group.

Taken together, common features of malignant pancreas cancer, i.e., high transaminase and TCA-cycle activity, are even more distinctive in cancers from patients who suffer from early recurrence after resection. Therefore, targeting these pathways might prolong survival of PDAC patients after surgery in the future.

\section{Conclusions}

In this study, we generated PDOs from early and late recurrent PDAC patients and uncovered metabolic alterations, which suggest an increased TCA-cycle activity and increased transamination in early recurrent PDOs. These are common features in malignant transformations of pancreatic tissue and their higher manifestation in early recurrent PDAC PDOs present a possible therapeutic vulnerability to fight pancreatic cancer relapse in the future.

Supplementary Materials: The following are available online at http://www.mdpi.com/2072-6694/12/6/1440/s1, Figure S1: Mutation allele frequencies of KRAS, Table S1: Primer sequences used in this study, Table S2: Antibodies used in this study, Table S3: Results of endometabolic profiling, Table S4: Results of exometabolic profiling.

Author Contributions: All authors have read and agree to the published version of the manuscript. Conceptualization, R.M.F., B.K., and U.A.W.; Formal analysis, L.M.B. and S.L.; Investigation, L.M.B., S.L., R.F.U.K., and S.H.; Methodology, L.M.B., R.F.U.K., and S.H.; Resources, R.M.F.; Supervision, B.K. and U.A.W.; Visualization, L.M.B., S.L., and S.H.; Writing—original draft, L.M.B. and S.L.; Writing-review and editing, R.M.F., B.K., and U.A.W.

Funding: The article processing charge was funded by the German Research Foundation (DFG) and the University of Freiburg in the funding program Open Access Publishing. R.F.U.K. and R.M.F. were funded by the Collaborative Research Center 850, project C09 (DFG), the German Cancer Consortium, and the Iten-Kohaut Foundation Zurich. S.H. is a recipient of a DGHO-GMIHO thesis fellowship.

Acknowledgments: We thank Silke Hempel, Stephanie Mewes and Marie-Claire Rittmann for excellent technical support and Olivia Sick and Julian Hipp for providing clinical data.

Conflicts of Interest: The authors declare no conflict of interest.

\section{References}

1. Muniraj, T.; Jamidar, P.A.; Aslanian, H.R. Pancreatic cancer: A comprehensive review and update. Dis. Mon. DM 2013, 59, 368-402. [CrossRef]

2. Ilic, M.; Ilic, I. Epidemiology of pancreatic cancer. World J. Gastroenterol. 2016, 22, 9694-9705. [CrossRef]

3. Heinemann, V. Gemcitabine: Progress in the treatment of pancreatic cancer. Oncology 2001, 60, 8-18. [CrossRef] [PubMed]

4. Kamisawa, T.; Wood, L.D.; Itoi, T.; Takaori, K. Pancreatic cancer. Lancet 2016, 388, 73-85. [CrossRef]

5. Kleeff, J.; Korc, M.; Apte, M.; la Vecchia, C.; Johnson, C.D.; Biankin, A.V.; Neale, R.E.; Tempero, M.; Tuveson, D.A.; Hruban, R.H.; et al. Pancreatic cancer. Nat. Rev. Dis. Prim. 2016, 2, 16022. [CrossRef] [PubMed]

6. Siegel, R.L.; Ma, J.; Zou, Z.; Jemal, A. Cancer statistics, 2014. CA A Cancer J. Clin. 2014, 64, 9-29. [CrossRef]

7. Rahib, L.; Smith, B.D.; Aizenberg, R.; Rosenzweig, A.B.; Fleshman, J.M.; Matrisian, L. Projecting Cancer Incidence and Deaths to 2030: The Unexpected Burden of Thyroid, Liver, and Pancreas Cancers in the United States. Cancer Res. 2014, 74, 2913-2921. [CrossRef]

8. Neoptolemos, J.P.; Kleeff, J.; Michl, P.; Costello, E.; Greenhalf, W.; Palmer, D.H. Therapeutic developments in pancreatic cancer: Current and future perspectives. Nat. Rev. Gastroenterol. Hepatol. 2018, 15, 333-348. [CrossRef]

9. SEER Cancer Statistics Review, 1975-2016. Available online: https://seer.cancer.gov/csr/1975_2016/index.html (accessed on 22 April 2020). 
10. Couch, F.J.; Johnson, M.R.; Rabe, K.G.; Brune, K.; De Andrade, M.; Goggins, M.; Rothenmund, H.; Gallinger, S.J.; Klein, A.; Petersen, G.M.; et al. The Prevalence of BRCA2 Mutations in Familial Pancreatic Cancer. Cancer Epidemiol. Biomark. Prev. 2007, 16, 342-346. [CrossRef]

11. Goldstein, A.M.; Fraser, M.C.; Hussussian, C.J.; Zametkin, D.P.; Fontaine, L.S.; Organic, S.M.; Dracopoli, N.C.; Struewing, J.; Ranade, K.; Clark, W.H.; et al. Increased Risk of Pancreatic Cancer in Melanoma-Prone Kindreds withp16INK4Mutations. N. Engl. J. Med. 1995, 333, 970-975. [CrossRef]

12. McWilliams, R.; Petersen, G.M.; Rabe, K.G.; Holtegaard, L.M.; Lynch, P.J.; Bishop, M.D.; Highsmith, W.E. Cystic fibrosis transmembrane conductance regulator (CFTR) gene mutations and risk for pancreatic adenocarcinoma. Cancer 2009, 116, 203-209. [CrossRef] [PubMed]

13. Iodice, S.; Gandini, S.; Maisonneuve, P.; Lowenfels, A.B. Tobacco and the risk of pancreatic cancer: A review and meta-analysis. Langenbecks Archiv für Chirurgie 2008, 393, 535-545. [CrossRef] [PubMed]

14. Hidalgo, M.; Cascinu, S.; Kleeff, J.; Labianca, R.; Löhr, J.-M.; Neoptolemos, J.P.; Real, F.X.; Van Laethem, J.-L.; Heinemann, V. Addressing the challenges of pancreatic cancer: Future directions for improving outcomes. Pancreatology 2015, 15, 8-18. [CrossRef] [PubMed]

15. Oberstein, P.E.; Olive, K.P. Pancreatic cancer: Why is it so hard to treat? Ther. Adv. Gastroenterol. 2013, 6, 321-337. [CrossRef] [PubMed]

16. Partensky, C. Toward a Better Understanding of Pancreatic Ductal Adenocarcinoma. Pancreas 2013, 42, 729-739. [CrossRef]

17. Maitra, A.; Hruban, R.H. Pancreatic Cancer. Annu. Rev. Pathol. 2008, 3, 157-188. [CrossRef]

18. Collisson, E.A.; Bailey, P.; Chang, D.K.; Biankin, A.V. Molecular subtypes of pancreatic cancer. Nat. Rev. Gastroenterol. Hepatol. 2019, 16, 207-220. [CrossRef]

19. Kamisawa, T.; Isawa, T.; Koike, M.; Tsuruta, K.; Okamoto, A. Hematogenous Metastases of Pancreatic Ductal Carcinoma. Pancreas 1995, 11, 345-349. [CrossRef]

20. Stathis, A.; Moore, M.J. Advanced pancreatic carcinoma: Current treatment and future challenges. Nat. Rev. Clin. Oncol. 2010, 7, 163-172. [CrossRef]

21. Gillen, S.; Schuster, T.; Büschenfelde, C.M.Z.; Friess, H.; Kleeff, J. Preoperative/Neoadjuvant Therapy in Pancreatic Cancer: A Systematic Review and Meta-analysis of Response and Resection Percentages. PLoS Med. 2010, 7, e1000267. [CrossRef]

22. Oettle, H.; Neuhaus, P.; Hochhaus, A.; Hartmann, J.T.; Gellert, K.; Ridwelski, K.; Niedergethmann, M.; Zülke, C.; Fahlke, J.; Arning, M.B.; et al. Adjuvant Chemotherapy With Gemcitabine and Long-term Outcomes Among Patients With Resected Pancreatic Cancer. JAMA 2013, 310, 1473. [CrossRef] [PubMed]

23. Schnelldorfer, T.; Ware, A.; Sarr, M.G.; Smyrk, T.C.; Zhang, L.; Qin, R.; Gullerud, R.E.; Donohue, J.H.; Nagorney, D.M.; Farnell, M.B. Long-Term Survival After Pancreatoduodenectomy for Pancreatic Adenocarcinoma. Ann. Surg. 2008, 247, 456-462. [CrossRef] [PubMed]

24. Iovanna, J.; Mallmann, M.C.; Goncalves, A.; Turrini, O.; Dagorn, J.-C. Current Knowledge on Pancreatic Cancer. Front. Oncol. 2012, 2. [CrossRef] [PubMed]

25. Hackert, T.; Büchler, M.W. Pancreatic Cancer: Advances in Treatment, Results and Limitations. Dig. Dis. 2013, 31, 51-56. [CrossRef] [PubMed]

26. Broeck, A.V.D.; Sergeant, G.; Ectors, N.; Van Steenbergen, W.; Aerts, R.; Topal, B. Patterns of recurrence after curative resection of pancreatic ductal adenocarcinoma. Eur. J. Surg. Oncol. 2009, 35, 600-604. [CrossRef] [PubMed]

27. Griffin, J.F.; Smalley, S.R.; Jewell, W.; Paradelo, J.C.; Reymond, R.D.; Hassanein, R.E.S.; Evans, R.G. Patterns of failure after curative resection of pancreatic carcinoma. Cancer 1990, 66, 56-61. [CrossRef]

28. Moletta, L.; Serafini, S.; Valmasoni, M.; Pierobon, E.S.; Ponzoni, A.; Sperti, C. Surgery for Recurrent Pancreatic Cancer: Is It Effective? Cancers 2019, 11, 991. [CrossRef]

29. Matsumoto, I.; Murakami, Y.; Shinzeki, M.; Asari, S.; Goto, T.; Tani, M.; Motoi, F.; Uemura, K.; Sho, M.; Satoi, S.; et al. Proposed preoperative risk factors for early recurrence in patients with resectable pancreatic ductal adenocarcinoma after surgical resection: A multi-center retrospective study. Pancreatology 2015, 15, 674-680. [CrossRef]

30. Kim, T.H.; Han, S.-S.; Park, S.-J.; Lee, W.J.; Woo, S.M.; Yoo, T.; Moon, S.H.; Kim, S.H.; Hong, E.K.; Kim, D.Y.; et al. CA 19-9 Level as Indicator of Early Distant Metastasis and Therapeutic Selection in Resected Pancreatic Cancer. Int. J. Radiat. Oncol. 2011, 81, 743-748. [CrossRef] 
31. Matsumoto, I.; Tanaka, M.; Shirakawa, S.; Shinzeki, M.; Toyama, H.; Asari, S.; Goto, T.; Yamashita, H.; Ishida, J.; Ajiki, T.; et al. Postoperative Serum Albumin Level is a Marker of Incomplete Adjuvant Chemotherapy in Patients with Pancreatic Ductal Adenocarcinoma. Ann. Surg. Oncol. 2014, 22, 2408-2415. [CrossRef]

32. Kang, C.M.; Kim, J.Y.; Choi, G.H.; Kim, K.S.; Choi, J.S.; Lee, W.J.; Kim, B.R. The Use of Adjusted Preoperative CA 19-9 to Predict the Recurrence of Resectable Pancreatic Cancer. J. Surg. Res. 2007, 140, 31-35. [CrossRef] [PubMed]

33. Tani, M.; Kawai, M.; Miyazawa, M.; Hirono, S.; Ina, S.; Nishioka, R.; Fujita, Y.; Uchiyama, K.; Yamaue, H. Liver metastasis as an initial recurrence has no impact on the survival of patients with resectable pancreatic adenocarcinoma. Langenbecks Archiv für Chirurgie 2008, 394, 249-253. [CrossRef] [PubMed]

34. Takahashi, H.; Ohigashi, H.; Ishikawa, O.; Gotoh, K.; Yamada, T.; Nagata, S.; Tomita, Y.; Eguchi, H.; Doki, Y.; Yano, M. Perineural Invasion and Lymph Node Involvement as Indicators of Surgical Outcome and Pattern of Recurrence in the Setting of Preoperative Gemcitabine-Based Chemoradiation Therapy for Resectable Pancreatic Cancer. Ann. Surg. 2012, 255, 95-102. [CrossRef] [PubMed]

35. Villarroel, M.C.; RajeshKumar, N.V.; Garrido-Laguna, I.; de Jesus-Acosta, A.; Jones, S.; Maitra, A.; Hruban, R.H.; Eshleman, J.R.; Klein, A.; Laheru, D.; et al. Personalizing cancer treatment in the age of global genomic analyses: PALB2 gene mutations and the response to DNA damaging agents in pancreatic cancer. Mol. Cancer Ther. 2010, 10, 3-8. [CrossRef]

36. Takai, E.; Yachida, S. Circulating tumor DNA as a liquid biopsy target for detection of pancreatic cancer. World J. Gastroenterol. 2016, 22, 8480-8488. [CrossRef]

37. Zhang, X.; Shi, S.; Zhang, B.; Ni, Q.; Yu, X.; Xu, J. Circulating biomarkers for early diagnosis of pancreatic cancer: Facts and hopes. Am. J. Cancer Res. 2018, 8, 332-353.

38. Lagies, S.; Schlimpert, M.; Braun, L.M.; Kather, M.; Plagge, J.; Erbes, T.; Wittel, U.A.; Kammerer, B. Unraveling altered RNA metabolism in pancreatic cancer cells by liquid-chromatography coupling to ion mobility mass spectrometry. Anal. Bioanal. Chem. 2019, 411, 6319-6328. [CrossRef]

39. Moreira, L.; Bakir, B.; Chatterji, P.; Dantes, Z.; Reichert, M.; Rustgi, A.K. Pancreas 3D Organoids: Current and Future Aspects as a Research Platform for Personalized Medicine in Pancreatic Cancer. Cell. Mol. Gastroenterol. Hepatol. 2017, 5, 289-298. [CrossRef]

40. Gadaleta, E.; Cutts, R.J.; Kelly, G.P.; Crnogorac-Jurcevic, T.; Kocher, H.; Lemoine, N.; Chelala, C. A global insight into a cancer transcriptional space using pancreatic data: Importance, findings, and flaws. Nucleic Acids Res. 2011, 39, 7900-79007. [CrossRef]

41. Lagies, S.; Schlimpert, M.; Neumann, S.; Wäldin, A.; Kammerer, B.; Borner, C.; Peintner, L. Cells grown in three-dimensional spheroids mirror in vivo metabolic response of epithelial cells. Commun. Boil. 2020, 3 , 1-10. [CrossRef]

42. Kim, M.P.; Evans, U.B.; Wang, H.; Abbruzzese, J.L.; Fleming, J.B.; E Gallick, G.; Abbrusseze, J.L. Generation of orthotopic and heterotopic human pancreatic cancer xenografts in immunodeficient mice. Nat. Protoc. 2009, 4, 1670-1680. [CrossRef] [PubMed]

43. Rubio-Viqueira, B.; Jimeno, A.; Cusatis, G.; Zhang, X.; Iacobuzio-Donahue, C.; Karikari, C.; Shi, C.; Danenberg, K.; Danenberg, P.V.; Kuramochi, H.; et al. An In vivo Platform for Translational Drug Development in Pancreatic Cancer. Clin. Cancer Res. 2006, 12, 4652-4661. [CrossRef] [PubMed]

44. Pérez-Mancera, P.A.; Guerra, C.; Barbacid, M.; Tuveson, D.A. What We Have Learned About Pancreatic Cancer from Mouse Models. Gastroenterology 2012, 142, 1079-1092. [CrossRef] [PubMed]

45. Boj, S.F.; Hwang, C.-I.; Baker, L.A.; Chio, I.I.C.; Engle, D.D.; Corbo, V.; Jager, M.; Ponz-Sarvise, M.; Tiriac, H.; Spector, M.S.; et al. Organoid models of human and mouse ductal pancreatic cancer. Cell 2014, 160, 324-338. [CrossRef]

46. Blokzijl, F.; de Ligt, J.; Jager, M.; Sasselli, V.; Roerink, S.; Sasaki, N.; Huch, M.; Boymans, S.; Kuijk, E.W.; Prins, P.; et al. Tissue-specific mutation accumulation in human adult stem cells during life. Nature 2016, 538, 260-264. [CrossRef]

47. Huch, M.; Gehart, H.; van Boxtel, R.; Hamer, K.; Blokzijl, F.; Verstegen, M.M.; Ellis, E.; van Wenum, M.; Fuchs, S.A.; de Ligt, J.; et al. Long-term culture of genome-stable bipotent stem cells from adult human liver. Cell 2014, 160, 299-312. [CrossRef]

48. Broutier, L.; Andersson-Rolf, A.; Hindley, C.J.; Boj, S.F.; Clevers, H.; Koo, B.-K.; Huch, M. Culture and establishment of self-renewing human and mouse adult liver and pancreas 3D organoids and their genetic manipulation. Nat. Protoc. 2016, 11, 1724-1743. [CrossRef] 
49. Baker, L.A.; Tiriac, H.; Tuveson, D.A. Generation and Culture of Human Pancreatic Ductal Adenocarcinoma Organoids from Resected Tumor Specimens. Adv. Struct. Saf. Stud. 2018, 97-115. [CrossRef]

50. Hussung, S.; Follo, M.; Klar, R.F.; Michalczyk, S.; Fritsch, K.; Nollmann, F.; Hipp, J.; Duyster, J.; Scherer, F.; Von Bubnoff, N.; et al. Development and Clinical Validation of Discriminatory Multitarget Digital Droplet PCR Assays for the Detection of Hot Spot KRAS and NRAS Mutations in Cell-Free DNA. J. Mol. Diagn. 2020. [CrossRef]

51. Lagies, S.; Pichler, R.; Kaminski, M.M.; Schlimpert, M.; Walz, G.; Lienkamp, S.; Kammerer, B. Metabolic characterization of directly reprogrammed renal tubular epithelial cells (iRECs). Sci. Rep. 2018, 8, 3878. [CrossRef]

52. Gradiz, R.; Silva, H.; Carvalho, L.; Botelho, M.F.; Mota-Pinto, A. MIA PaCa-2, and PANC-1—Pancreas ductal adenocarcinoma cell lines with neuroendocrine differentiation and somatostatin receptors. Sci. Rep. 2016, 6, 21648. [CrossRef] [PubMed]

53. Dufour, E.; Gay, F.; Aguera, K.; Scoazec, J.-Y.; Horand, F.; Lorenzi, P.L.; Godfrin, Y. Pancreatic Tumor Sensitivity to Plasma L-Asparagine Starvation. Pancreas 2012, 41, 940-948. [CrossRef] [PubMed]

54. Viale, A.; Pettazzoni, P.; Lyssiotis, C.A.; Ying, H.; Sanchez, N.; Marchesini, M.; Carugo, A.; Green, T.; Seth, S.; Giuliani, V.; et al. Oncogene ablation-resistant pancreatic cancer cells depend on mitochondrial function. Nature 2014, 514, 628-632. [CrossRef]

55. Kamphorst, J.J.; Nofal, M.; Commisso, C.; Hackett, S.R.; Lu, W.; Grabocka, E.; Heiden, M.G.V.; Miller, G.; Drebin, J.A.; Bar-Sagi, D.; et al. Human pancreatic cancer tumors are nutrient poor and tumor cells actively scavenge extracellular protein. Cancer Res. 2015, 75, 544-553. [CrossRef] [PubMed]

56. Hui, S.; Ghergurovich, J.M.; Morscher, R.J.; Jang, C.; Teng, X.; Lu, W.; Esparza, L.A.; Reya, T.; Zhan, L.; Guo, J.Y.; et al. Glucose feeds the TCA cycle via circulating lactate. Nature 2017, 551, 115-118. [CrossRef] [PubMed]

57. Li, J.-T.; Yin, M.; Wang, D.; Wang, J.; Lei, M.-Z.; Zhang, Y.; Liu, Y.; Zhang, L.; Zou, S.-W.; Hu, L.-P.; et al. BCAT2-mediated BCAA catabolism is critical for development of pancreatic ductal adenocarcinoma. Nature 2020, 22, 167-174. [CrossRef] [PubMed]

58. Mayers, J.; Torrence, M.E.; Danai, L.V.; Papagiannakopoulos, T.; Davidson, S.M.; Bauer, M.R.; Lau, A.N.; Ji, B.W.; Dixit, P.D.; Hosios, A.M.; et al. Tissue of origin dictates branched-chain amino acid metabolism in mutant Kras-driven cancers. Science 2016, 353, 1161-1165. [CrossRef] [PubMed] 